\title{
Nuevos registros de Gentianaceae y Lentibulariaceae para Honduras
}

\author{
New records of Gentianaceae and Lentibulariaceae from Honduras
}

\author{
Hermes Vega ${ }^{1}$, Delmer J. Hernández ${ }^{2}$, William Cetzal-Ix ${ }^{3,7}$, Katya Romero-Soler ${ }^{4}$, Mario Solis ${ }^{5}$ \& Edgar Mó ${ }^{6}$
}

\begin{abstract}
Resumen
Se presentan nuevos registros de Gentianaceae y Lentibulariaceae para la flora de Honduras. Utricularia endresii de los departamentos Atlántida, Comayagua y Olancho, U. jamesoniana (Lentibulariaceae) de los departamentos Atlántida, Comayagua, Gracias a Dios y Olancho y Voyria truncata (Gentaniaceae) del departamento Comayagua. Además, se proveen ilustraciones para las especies y un mapa de distribución. Palabras clave: áreas naturales protegidas, florística, plantas carnívoras, saprofitas.
\end{abstract}

\begin{abstract}
New records of Gentianaceae and Lentibulariaceae for the Hondurean flora are presented. Utricularia endresii of the departments of Atlántida, Comayagua and Olancho, U. jamesoniana (Lentibulariaceae) of the departments of Atlántida, Comayagua, Gracias a Dios and Olancho, and Voyria truncata (Gentaniaceae) of the department of Comayagua. Furthermore, illustrations for the species and a distribution map are provided.
\end{abstract}

Key words: natural protected areas, floristic, carnivorous plants, saprophytes.

\section{Introducción}

Honduras es un país Centroamericano con una gran diversidad florística poco documentada, como consecuencia de la escasez de taxónomos vegetales. Frecuentemente son registrados nuevos hallazgos de plantas para el país, sin embargo, parte de esta información no es publicada formalmente con especímenes de respaldo que permitan corroborar su presencia. Por consiguiente, en revisiones sistemáticas o base de datos internacionales (e.g., <http:// tropicos.org $>$ ) es escasa la información sobre la biodiversidad de Honduras. Por ejemplo, en revisiones taxonómicas para algunas especies centroamericanas o de amplia distribución usualmente se indican los puntos extremos de sus distribuciones y los países donde se encuentran presentes, pero en los casos cuando no hay especímenes de respaldo se señala para el país como una especie "esperada". En este sentido, para Honduras lo constituye algunas especies de las familias Gentianaceae y Lentibulariaceae que han sido registrados en países vecinos.

Gentianaceae es una familia con distribución cosmopolita, incluye 1,651 especies en 86 géneros (Sánchez-González 2010). Honduras posee 19 especies en siete géneros: Centaurium Hill (6), Curtia Cham. \& Schltdl. (1), Halenia Borkh. (2), Irlbachia Mart. (1), Lisianthius P.Browne (2), Schultesia Mart. (3) y Voyria

\footnotetext{
${ }^{1}$ MAPANCE, Mancomunidad de Municipios del Parque Nacional Celaque, Bo. Mercedes, Edif. de Gobernación, Gracias, 42101, Lempira, Honduras.

${ }^{2}$ Grupo de Investigación de Biodiversidad de Honduras BALAM (GIBH-BALAM), Tegucigalpa, 11101, Francisco Morazán, Honduras.

${ }^{3}$ Instituto Tecnológico de Chiná, Calle 11 entre 22 y 28, Colonia Centro Chiná 24050, Campeche, México.

${ }^{4}$ Herbario CICY, Centro de Investigación Científica de Yucatán, A.C. (CICY), Calle 43 130. Col. Chuburná de Hidalgo, Mérida 97200 , Yucatán, México.

${ }^{5}$ Escuela de Biología, Universidad Nacional Autónomas de Honduras (UNAH), Tegucigalpa, 11101, Francisco Morazán, Honduras.

${ }^{6}$ Orquideario Agronomía-CUNOR-USAC, Centro Universitario del Norte, Universidad de San Carlos de Guatemala, Cobán, 16001, Alta Verapaz, Guatemala.

${ }^{7}$ Autor para la correspondencia: rolito22@hotmail.com
} 
Aubl. (4) (Nelson 2008). En lo que respecta a Voyria, se distribuye principalmente en los Trópicos Americanos con 19 especies y una en el oeste de África tropical (Maas \& Ruyters 1986). En Honduras, se encuentra registrado $V$. alba (Standl.) L.O.Williams, V. aphylla (Jacq.) Pers., V. parasitica (Schltdl. et Cham.) Ruyters \& Maas, V. tenella Hook. (Nelson 2008; Wilbur 2015); estas especies son de bosques húmedos y pluviales y de elevaciones de $0-1,700 \mathrm{~m}$; caracterizándose por plantas micoheterotróficas, sin clorofila, con hojas opuestas, reducidas y escamiformes. No obstante, suelen confundirse con otras micoheterotróficas de la familia Burmanniaceae, pero se diferencian por las hojas alternas y flores 3 ó 6 meras y ovario ínfero (Maas \& Ruyters 1986).

En cuanto a Lentibulariaceae, es una familia de distribución cosmopolita (de regiones templadas, tropicales y árticas), conformada por más de 300 especies, incluye plantas herbáceas, anuales o perennes, que pueden ser terrestres, acuáticas o epífitas (Taylor 1989; Zamudio 1990). Honduras posee 17 especies en tres géneros (Genlisea A. St.-Hil., Pinguicula L. y Utricularia L.), siendo el más numeroso Utricularia con 12 especies registradas (Nelson 2008); este género de 214 especies incluye plantas carnívoras que viven en agua dulce y en suelos húmedos en todos los continentes con excepción de la Antártida (Taylor 1989). Los miembros acuáticos del género presentan vejigas grandes y conspicuas (Salmon 2001), mientras que las especies epífitas pueden crecer en musgos húmedos y en cortezas esponjosas de árboles en selvas húmedas o lluviosas (Lloyd 1942). Todas sus especies son consideradas carnívoras, debido a que capturan pequeños organismos por medio de trampas con vejigas de succión (Rutishauser \& Isler 2001).

Como parte de los estudios florísticos realizados por los herbarios EAP y TEFH y como un esfuerzo por contribuir al conocimiento de la flora de Honduras, se realizaron viajes de exploración y recolección botánica en diversos departamentos del país (Atlántida, Comayagua, Gracias a Dios y Olancho). Durante este trabajo se encontraron especies no registradas para la flora de Honduras.

\section{Materiales y Métodos}

Los especímenes de respaldo fueron fotografiados en su hábitat (Figs. 1-3), herborizados y depositados en los herbarios de la Universidad Nacional Autónoma de Honduras (TEFH) y Paul C. Standley de la Escuela Agrícola Zamorano (EAP). Para las identificaciones se usaron claves taxonómicas de Flora mesoamericana.

\section{Resultados y Discusión}

\section{Gentianaceae}

1. Voyria truncata (Standl.) Standl. \& Steyerm., Publ. Field Mus. Nat. Hist., Bot. Ser. 23: 78. 1944. Leiphaimos truncata Standl., Contr. U.S. Natl. Herb. 20: 196. 1919. Tipo: PANAMÁ. Pittier $4306 a$ (Holotipo: US). $\quad$ Fig. 1

Hierbas de hasta $7 \mathrm{~cm}$ alto; tallos simples, purpúreos a blancos, sin hojas evidentes. Inflorescencia de una flor solitaria; sin brácteas; pedicelos 5-10 mm largo. Flores 5-6 meras; cáliz 5-7 mm largo, corola 15-25 mm largo, puberulenta, el tubo 20-35 mm largo, purpúreo a blanco, cilíndrico, los lobos 10-20 × 0,5 $\mathrm{mm}$, púrpura-rosados a blancos, angostamente elípticos a ovados-triangulares. Ovario ca. 4,5 $\times 1,8 \mathrm{~mm}$, cilíndrico, sésil, truncado. Cápsula no vista.

Material examinado: Comayagua, Parque Nacional Cerro Azul Meámbar, Sendero Los Vencejos, bosque lluvioso tropical, 800 m, 28.I.2014, J. Hernández \& H. Vega 203 (TEFH).

Voyria truncata se encuentra registrada en Belice, Guatemala, Nicaragua, Costa Rica, Panamá, Colombia y Perú. Crece en selvas altas perennifolias y bosques lluviosos tropicales, desde el nivel de mar hasta los 1,400 m (Maas \& Ruyters 1986). La colección de Honduras se recolectó en el departamento de Comayagua, aproximadamente entre 140 y 300 $\mathrm{km}$ de distancia de las poblaciones conocidas de Guatemala y Nicaragua, respectivamente (Fig. 4). Los individuos de la población de Comayagua, crecen sobre materia orgánica en descomposición, en bosque lluvioso subtropical, a 800 m de elevación. Por su distribución amplia no ha sido considerada bajo alguna categoría de riesgo de conservación. La población del Parque Nacional Cerro Azul Meámbar se encuentra protegida dentro de la zona de amortiguamiento, por lo que su conservación en Honduras no se encuentra bajo riesgo. Morfológicamente, Voyria truncata se diferencia de otras especies registradas en el país por las flores púrpura blanquecinas con los lobos del cáliz elípticos a ovado-triangulares (Fig. 1). 

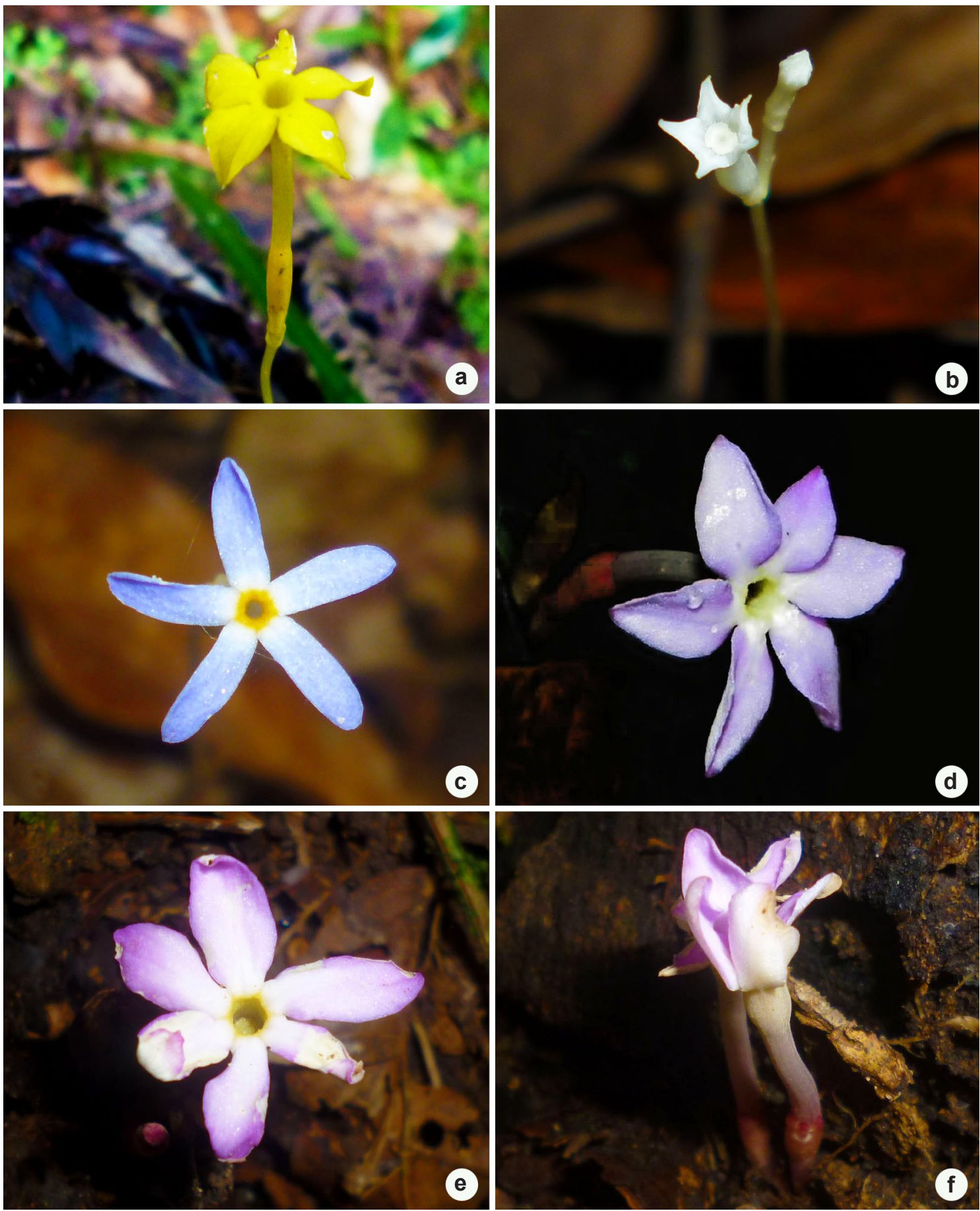

Figura 1 - Algunas especies de Voyrias de Honduras - a. Voyria aphylla; b. Voyria parasitica; c. Voyria tenella; d-f. Voyria truncata - d. flor en vista lateral; e. flor en vista frontal; f. hábito.

Figure 1 - Some species of Voyrias of Honduras - a. Voyria aphylla; b. Voyria parasitica; c. Voyria tenella; d-f. Voyria truncata - d. flower in lateral view; e. flower in front view; f. habit. 


\section{Lentibulariaceae}

2. Utricularia endresii Rchb.f., Gard. Chron., n.s. 1874(2): 582. 1874. Orchyllium endresii (Rchb.f.) Barnhart ex MacIntyre \& Chrysler, Bull. Torrey Bot. Club 70: 252. 1943. Tipo: COSTA RICA. $2000 \mathrm{ft}$. alt., in woods, on trunks, and on the soil at rather dry stations, Endres s.n. (Lectotipo: W-1889-286465, Isolectotipo: K-K000779804).

Fig. 2

Planta perenne, epífita. Hojas 4-6 × 1,3-1,6 $\mathrm{cm}$, oblongo-elípticas, membranáceas, verdes, las láminas mucho más largas que los pecíolos. Racimos 4,5-6,5 cm largo, pedicelos 2,5-2,8 cm largo; lóbulos del cáliz 1,1-1,5 cm largo, similares, ovados; corola 1,2-1,5 cm largo, lila blanquecina con una mancha amarilla, el labio inferior transversalmente elíptico y con el ápice 3-crenado. Cápsula no vista.

Material examinado: Atlántida, Tela, Refugio de Vida Silvestre Texíguat, Camino de la Laguna El Chino, La Liberación, bosque nuboso, 15²9'35.91'N, 87¹8'26.31'O, 1,600 m, 7.VII.2010, P. House \& H. Vega 4876 (TEFH); Ceiba, Parque Nacional Pico Bonito, 15³9'31.40”N, 8652'13.22”O, IX.2010, M. Solis 1 (TEFH-Foto); Comayagua, Parque Nacional Cerro Azul Meámbar (PANACAM), Cerro Aguacatales cercano a la Aldea El Palmital, bosque nuboso, 1449'41.19'N, 8750'53.71'O, 1,540 m, 17.XI.2013, J. Hernández 204 (TEFH); Gracias a Dios, Cerro Baltimore, montañas cercanas a Las Marías, Biosfera del Río Plátano, bosque nuboso, 1543'53.76”N, 8454'1.01”O, 820 m, 11.VI.2012, J. Hernández 201 (EAP); Olancho, Parque Nacional Patuca, punto más elevado de la Montaña "Entre Ríos" bosque nuboso, $14^{\circ} 10^{\prime} 43.95^{\prime}$ N , 85³9'26.22”O, 1,500 m, 1.VI.2013, K. Soler 818 (EAP).

Utricularia endresii se ha registrado en Nicaragua, Costa Rica, Panamá, Colombia, Ecuador y Venezuela. En Nicaragua, recientemente fue registrado por Pérez-Soto \& López-Saéz (2009) de los volcanes Concepción y Maderas en la Isla de Ometepe. En general, crece como epífita sobre troncos y ramas de árboles u ocasionalmente como epipétrica en taludes asociadas a musgos, en bosques nublados o lluviosos, desde el nivel del mar hasta los 2,500 m (<http://tropicos. org $>2015)$. Las colecciones de Honduras se recolectaron aproximadamente entre 320 y 490 $\mathrm{km}$ de distancia de las poblaciones conocidas de Nicaragua y Costa Rica, respectivamente, lo que incrementa significativamente su distribución. Las colecciones provienen de los departamentos Atlántida, Comayagua, Gracias a Dios y Olancho, en bosque nuboso (bosque tropical siempreverde mixto altimontano), entre (820-)1,540-1,600 m (Fig. 4). Por su distribución amplia no ha sido considerada bajo alguna categoría de riesgo de conservación. Las cuatro poblaciones aquí registradas se encuentran protegidas dentro de las zonas de amortiguamiento y núcleo de las áreas naturales protegidas: Biosfera del Río Plátano, Parque Nacional Pico Bonito, Parque Nacional Patuca y El Refugio de Vida Silvestre Texíguat.

3. Utricularia jamesoniana Oliv., J. Proc. Linn. Soc., Bot. 4: 169-170, t. 1, f. 1. 1860. Tipo: ECUADOR. Napo: Consanga River, halfway Archedonae, prov. Ecuador, W. Jameson 1 (Holotipo: K).

Utricularia schimperi Schenk, Jahrb. Wiss. Bot. 18: 230-232. 1887. Orchyllium schimperi (Schenk) Barnhart, Bull. Torrey Bot. Club 58: 469. 1931. Tipo: DOMINICA. A.F. Schimper s.n. (Holotipo: B, destruido).

Utricularia verapazensis Morong ex Donn. Sm., Bot. Gaz. 18(6): 209. 1893. Tipo: GUATEMALA. Alta Verapaz: tree trunks near San Pedro Carchá, alt. $3000 \mathrm{ft}, \mathrm{I} .1887$, H. von Tuerckheim 1109 (Holotipo: US).

Fig. 3

Planta perenne, epífita. Hojas $1,5-2,2 \times$ 0,3-0,6 mm, obovadas. Racimos 2,2-2,5 cm largo, pedicelos 0,5-1,2 mm largo; lóbulos del cáliz 0,8-1,2 mm largo, similares, ampliamente ovados; corola 0,6-1,1 mm largo, blanca matizada de púrpura y con una mancha amarilla, estipitadoglandular. Cápsula 0,5-1,0 mm largo, oblongoelipsoide, ventralmente 1-valvada.

Material examinado: Atlántida, Tela, Refugio de Vida Silvestre Texíguat, Camino de la Laguna El Chino, La Liberación, bosque lluvioso, 15²9'35.91'N, 87¹8'26.31'O, 1,438 m, 7.VII.2010, P. House \& H. Vega 4884 (TEFH); Comayagua, Parque Nacional Cerro Azul Meámbar (PANACAM), Los Pinos, cerca del área de senderos, bosque lluvioso, 14 ${ }^{\circ} 52^{\prime} 19.36$ ”N, 8754'2.26”'O, 1,200 m, 28.I.2014, J. Hernández 206 (TEFH); Olancho, Parque Nacional Patuca, Montaña “Entre Ríos” bosque lluvioso, 149'52.43”N, 8540'6.11”'O, 1,300 m, 29.VI.2013, K. Soler 815 (EAP).

Esta especie se ha registrado en México (Chiapas), Centroamérica (Guatemala, Costa Rica, Nicaragua, Panamá,), Sudamérica (Colombia, Venezuela, Guyana, Guayana Francesa, Surinam, Brasil, Ecuador, Perú, Bolivia) y las Antillas (Martinica, Dominica, Guadalupe, Republica Dominicana) (Olvera 1996; <http://tropicos. org $>2015)$. En general, crece sobre troncos $y$ ramas, asociada a musgos en bosques nublados o lluviosos, a 1,700-2,500 m (Olvera 1996). Las 

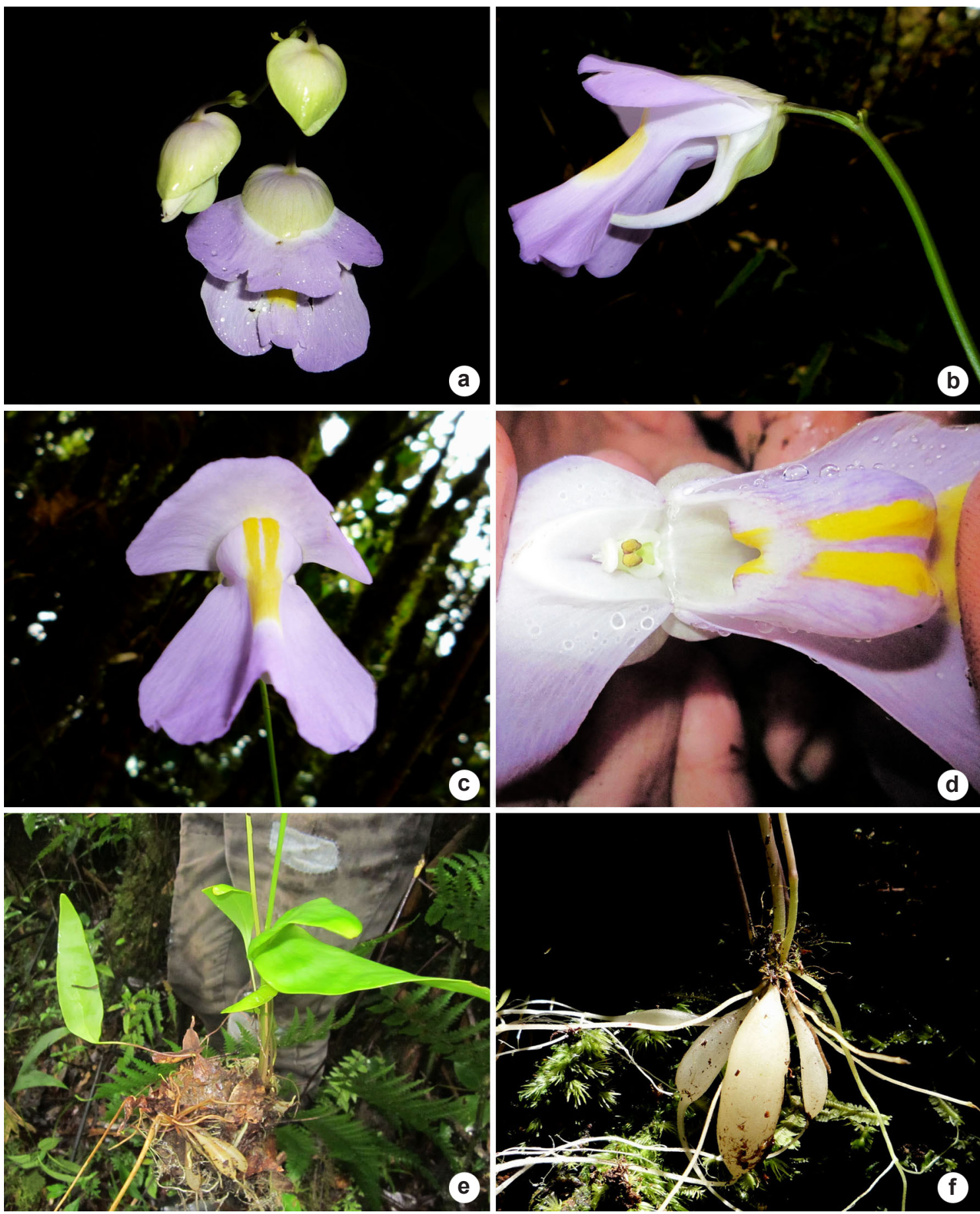

Figura 2 - Utricularia endresii - a. flor, vista frontal; b. flor, vista lateral; c. detalle de la flor, vista frontal; d. detalle de los estambres; e. detalle de las hojas; f. vista del sistema radical, tubérculos.

Figure 2 - Utricularia endresii - a. flower, front view; b. flower, lateral view; c. detail of the flower, frontal view; d. detail of the stamens; e. detail of the leaves; f. view of the root system, tubers. 

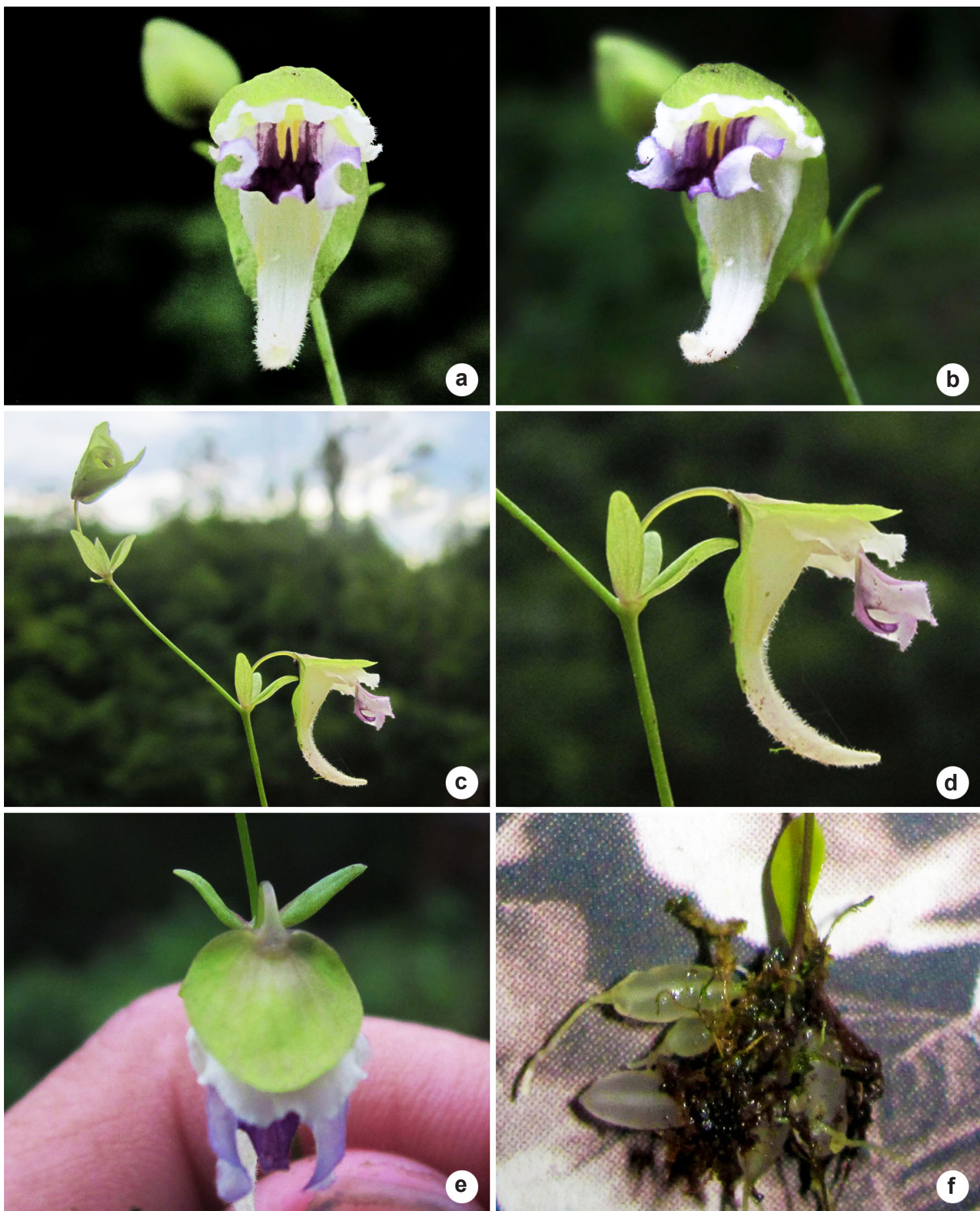

Figura 3 - Utricularia jamesoniana - a. flor, vista frontal; b. flor, vista lateral; c. inflorescencia; d. detalle de la flor, vista lateral; e. sépalo, vista anterior; f. vista del sistema radical, tubérculos.

Figure 3 - Utricularia jamesoniana - a. flower, front view; b. flower, lateral view; c. inflorescence; d. detail of flower, lateral view; e. sepal, frontal view; f. view of the root system, tubers. 


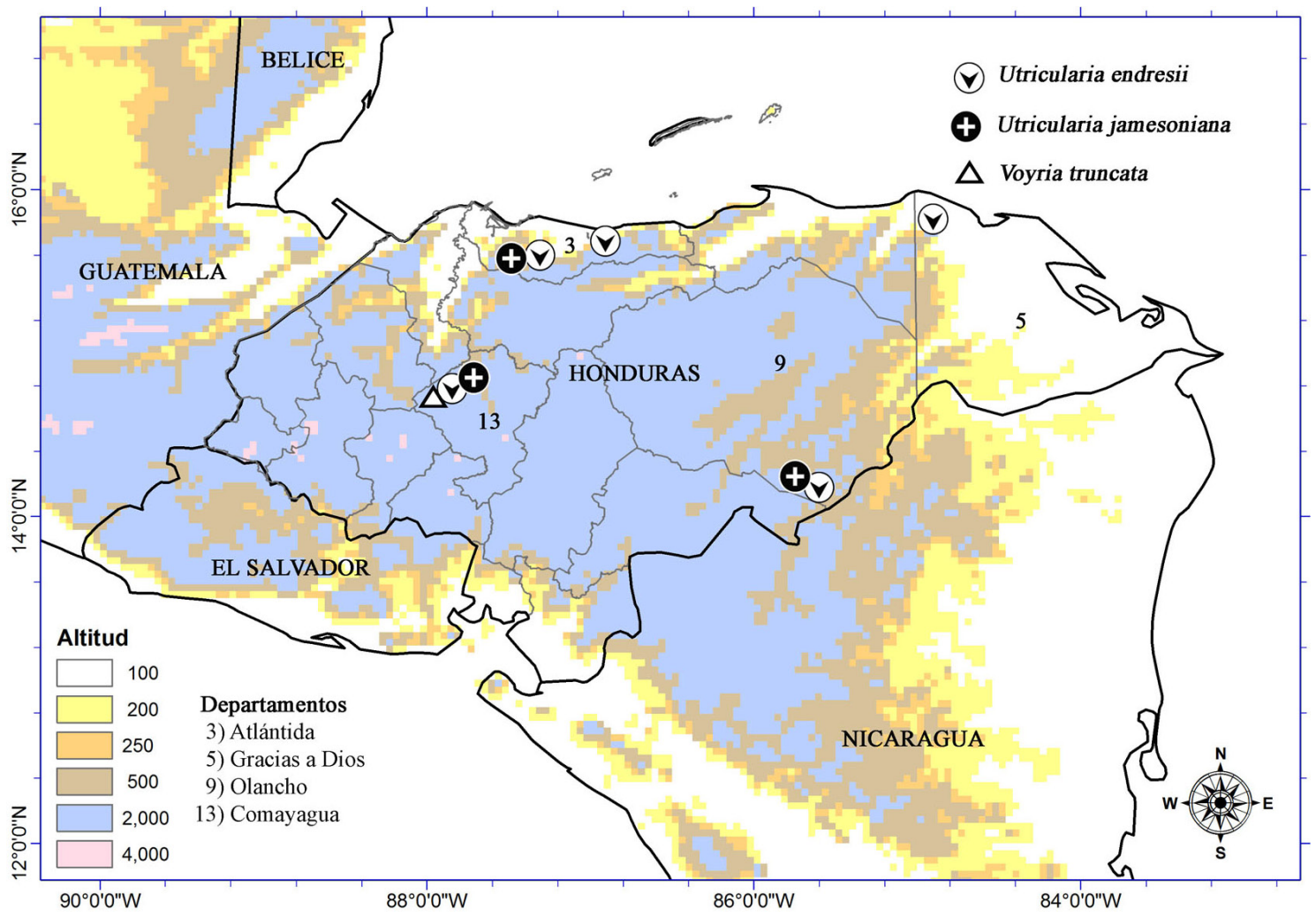

Figura 4 - Distribución de los nuevos registros en Honduras.

Figure 4 - Distribution of new records in Honduras.

colecciones aquí registradas de Honduras se encontraron aproximadamente entre 250 y 100 $\mathrm{km}$ de distancia de las poblaciones conocidas de Guatemala y Nicaragua, respectivamente. Estas colecciones provienen de los departamentos Atlántida, Comayagua y Olancho, en bosque lluvioso, entre 1,200-1,438 m. Por su distribución amplia no ha sido considerada bajo alguna categoría de riesgo de conservación. Las tres poblaciones aquí registradas se encuentran protegidas dentro las zonas de amortiguamiento y núcleo de las áreas naturales protegidas: Parque Nacional Cerro Azul Meámbar, Parque Nacional Patuca y El Refugio de Vida Silvestre Texíguat.

En Honduras, Utricularia endresi y $U$. jamesoniana son especies de amplia distribución en la vertiente Atlántica, registradas desde la porción sur en el departamento de Atlántida y en la porción central en el departamento de Comayagua, hasta la porción oriente en el departamento de Olancho. Posiblemente se encuentran en otros departamentos del país en parques nacionales y reservas intermedias entre ellas, a elevaciones entre 800 y $1,700 \mathrm{~m}$. Sin embargo, estas especies crecen de manera alopátrica, la primera en bosques nubosos a elevaciones entre (820-) $1,540-1,600 \mathrm{~m}$ y la segunda en bosques lluviosos tropicales a elevaciones entre 1,200-1,438 m. Morfológicamente, Utricularia endresii se caracteriza por plantas con hojas largas y anchas (4-6 × 1,3-1,6 cm), inflorescencias con pedicelos largos $(2,5-2,8 \mathrm{~cm}$ largo) y flores lilas blanquecinas con una mancha amarilla; en contraste, $U$. jamesoniana se caracteriza por plantas con hojas más pequeñas y delgadas $(1,5-2,2 \times 0,3-0,6 \mathrm{~mm})$, obovadas, inflorescencias con pedicelos cortos $(0,5-1,2 \mathrm{~mm}$ largo $)$ y flores blancas matizadas de púrpura y con una mancha amarilla.

\section{Agradecimientos}

Cyril Nelson, por la determinación de Voyria truncata. La cuarta autora agradece al CONACYT, por la beca otorgada para estudios de maestría. Eliana Noguera Savelli, por sus comentarios y sugerencias que ayudaron a mejorar el manuscrito. 


\section{Referencias}

Lloyd, F.E. 1942. The Carnivorous plants. Chronica Botanica, New York. 352p.

Maas, P.J.M. \& Ruyters, P. 1986. Voyria and Voyriella (Saprophytic Gentianaceae). Flora Neotropica 41: 1-93.

Nelson, C. 2008. Catálogo de plantas vasculares de Honduras, Spermatophytas. Editorial Guaymuras, Tegucigalpa. $1576 \mathrm{p}$.

Olvera, M. 1996. El género Utricularia (Lentibulariaceae) en México. Anales del Instituto de Biología, Universidad Nacional Autónoma de México. Serie botánica 67: 347-384.

Pérez-Soto, J. \& López-Saéz, J.A. 2009. Estudio preliminar de las orquídeas de la Isla de Ometepe. Revista Universidad y Ciencia, UNAN-Managua 4: 1-20.

Rutishauser, R. \& Isler, B. 2001. Developmental genetics and morphological evolution of flowering plants, especially Bladderworts (Utricularia): fuzzy arberian morphology complements classical morphology. Annals of Botany 88: 1173-1202.
Salmon, B. 2001. Carnivorous plants of New Zealand. Ecosphere publications, Auckland. 303p.

Sánchez-González, J. 2010. Gentianaceae. In: Hammel B.E.; Grayum, M.H.; Herrera, C. \& Zamora, N. (eds.). Manual de plantas de Costa Rica, Vol. V (Clusiaceae-Gunneraceae). Monographs in Systematic Botany from the Missouri Botanical Garden 119: 821-840.

Taylor, P. 1989. The genus Utricularia - a taxonomic monograph. Royal Botanic Gardens, London. $724 \mathrm{p}$.

Tropicos.org. 2015. Missouri Botanical Garden. Disponible en <http://www.tropicos.org/ Name/18300113>. Acceso a 10 Noviembre 2015.

Wilbur, R.L. 2015. 15. Voyria Aubl. Flora Mesoamericana 4: 658. Disponible en $<\mathrm{http} / /$ www.tropicos.org/Name/40025027? projectid=3>. Acceso a 10 Noviembre 2015.

Zamudio, S. 1990. El género Pinguicula (Lentibulariaceae) en México. Resúmenes del V Congreso Latinoamericano de Botánicas, La Habana. 399p. 